\title{
On the thermal sensitivity of binary formation in collapsing molecular clouds
}

\author{
R. Riaz, ${ }^{1 \star}$ S. Z. Farooqui ${ }^{2}$ and S. Vanaverbeke ${ }^{3,4}$ \\ ${ }^{1}$ Institute of Space and Planetary Astrophysics (ISPA), University of Karachi, Karachi 75270, Pakistan \\ ${ }^{2}$ Faculty of Engineering Sciences, National University of Science Technology, Islamabad 46000, Pakistan \\ ${ }^{3}$ Centre for Mathematical Plasma-Astrophysics, Department of Mathematics, KU Leuven, Celestijnenlaan 200B, B-3001 Heverlee, Belgium \\ ${ }^{4}$ Katholieke Universiteit Leuven Campus Kortrijk, E.-Sabbelaan 53, B-8500 Kortrijk, Belgium
}

Accepted 2014 July 24. Received 2014 July 4; in original form 2014 March 21

\begin{abstract}
We report the results of a numerical study on the initial formation stages of low-mass protostellar binary systems. We determine the separation of protostellar binaries formed as a function of the initial thermal state by varying the initial temperature in a slightly modified version of the Burkert and Bodenheimer collapse test. We find that the outcome is highly sensitive to both the initial temperature of the cloud and the initial amplitude of azimuthal density perturbation $A$. For $A=10$ per cent, variations of only $1 \mathrm{~K}$ below $10 \mathrm{~K}$ lead to changes of up to 100 au (i.e. of the order of 30 per cent) in the instantaneous separation, whereas for this small $A$ the initial temperatures above $10 \mathrm{~K}$ yield, instead of a binary, a single low-mass fragment that never reaches protostellar densities. Protostellar binaries, however, do emerge when the perturbation amplitude is increased from 10 to 25 per cent. We also investigate the impact of the critical density which governs the transition from isothermal to adiabatic thermodynamic behaviour of the collapsing gas. We find that the critical density not only affects the overall structural evolution of the gas envelope, but also the size of the rotating disc structures formed during collapse as well as the number of protostellar fragments resulting from the final fragmentation of the discs. This mechanism can give rise to young protostellar objects constituting bound multiple stellar systems.
\end{abstract}

Key words: binaries: general-brown dwarfs-stars: early-type-stars: formation-stars: low-mass.

\section{INTRODUCTION}

Molecular clouds are believed to be among the coolest regions in a galaxy where low-mass star formation can take place. Because star formation involves an enormous range of complex physical processes, it is difficult to construct exhaustive theoretical models which include every aspect of the problem (Krumholtz 2011). It is generally found that systems consisting of multiple protostars emerge as a result of the gravitational collapse of rotating molecular cloud cores (Raghavan et al. 2010; Janson et al. 2012). A significant fraction of these multiple star systems are binary systems. In fact, infrared surveys of nearby star formation regions reveal that around 75 per cent of the newly formed protostars are part of multiple stellar systems (Duchêne et al. 2007). Furthermore, an interesting population of loosely bound very low mass binaries with large separation $d(d>100 \mathrm{au})$ is also found in very young star-forming regions (e.g. Luhman 2004; Bouy et al. 2006; Béjar et al. 2008).

^E-mail: rafilriaz@yahoo.com
Theories which try to explain the formation processes of binary star systems include disc fragmentation, fission of a protostar(s), and dynamical capture of protostars into bound systems (Moeckel $\&$ Bally 2007; Tobin et al. 2013). Among these possible scenarios, the disc fragmentation model is the subject of the investigation reported in this paper. The basic purpose of this paper is to reexamine numerically certain aspects of the gravitational collapse and the subsequent fragmentation of rotating molecular cloud cores. We focus on the dependence of disc fragmentation as a low-mass binary and multiple star formation mechanism on the initial temperature as well as the critical density which marks the transition from isothermal to adiabatic thermodynamic behaviour of the collapsing gas.

A significant number of simulation results have so far been reported in the literature in which binary systems emerging from disc fragmentation have been investigated (Fumitaka \& Li 2003; Machida et al. 2008; Forgan \& Rice 2012). The availability of advanced computational resources and improved versions of stateof-the-art star formation codes have significantly contributed to our 
Table 1. Summary of the physical parameters and the final outcome for the models considered in this paper. The initial mass, radius and density for each model are given by the constant values $5 \times 10^{16} \mathrm{~cm}$, $1 \mathrm{M}_{\odot}$, and $3.8 \times 10^{-18} \mathrm{~g} \mathrm{~cm}^{-3}$, respectively.

\begin{tabular}{lccccc}
\hline Model & Temperature $(\mathrm{K})$ & $c_{0}\left(\mathrm{~cm} \mathrm{~s}^{-1}\right)$ & $\rho_{\text {crit }}\left(\mathrm{g} \mathrm{cm}^{-3}\right)$ & Final outcome & Binary separation (au) \\
\hline A & 8 & 1480.0 & $5 \times 10^{-14}$ & Binary & 278.685 \\
B & 9 & 1570.0 & $5 \times 10^{-14}$ & Binary & 343.235 \\
C & 10 & 1650.0 & $5 \times 10^{-14}$ & Binary & 378.364 \\
D & 11 & 1730.0 & $5 \times 10^{-14}$ & Single & - \\
E & 12 & 1810.0 & $5 \times 10^{-14}$ & Single & - \\
F & 10 & 1650.0 & $5 \times 10^{-15}$ & None & - \\
G & 10 & 1650.0 & $5 \times 10^{-13}$ & Triple & - \\
H & 11 & 1730.0 & $5 \times 10^{-14}$ & Binary & 228.438 \\
I & 12 & 1810.0 & $5 \times 10^{-14}$ & Binary & 197.608 \\
\hline
\end{tabular}

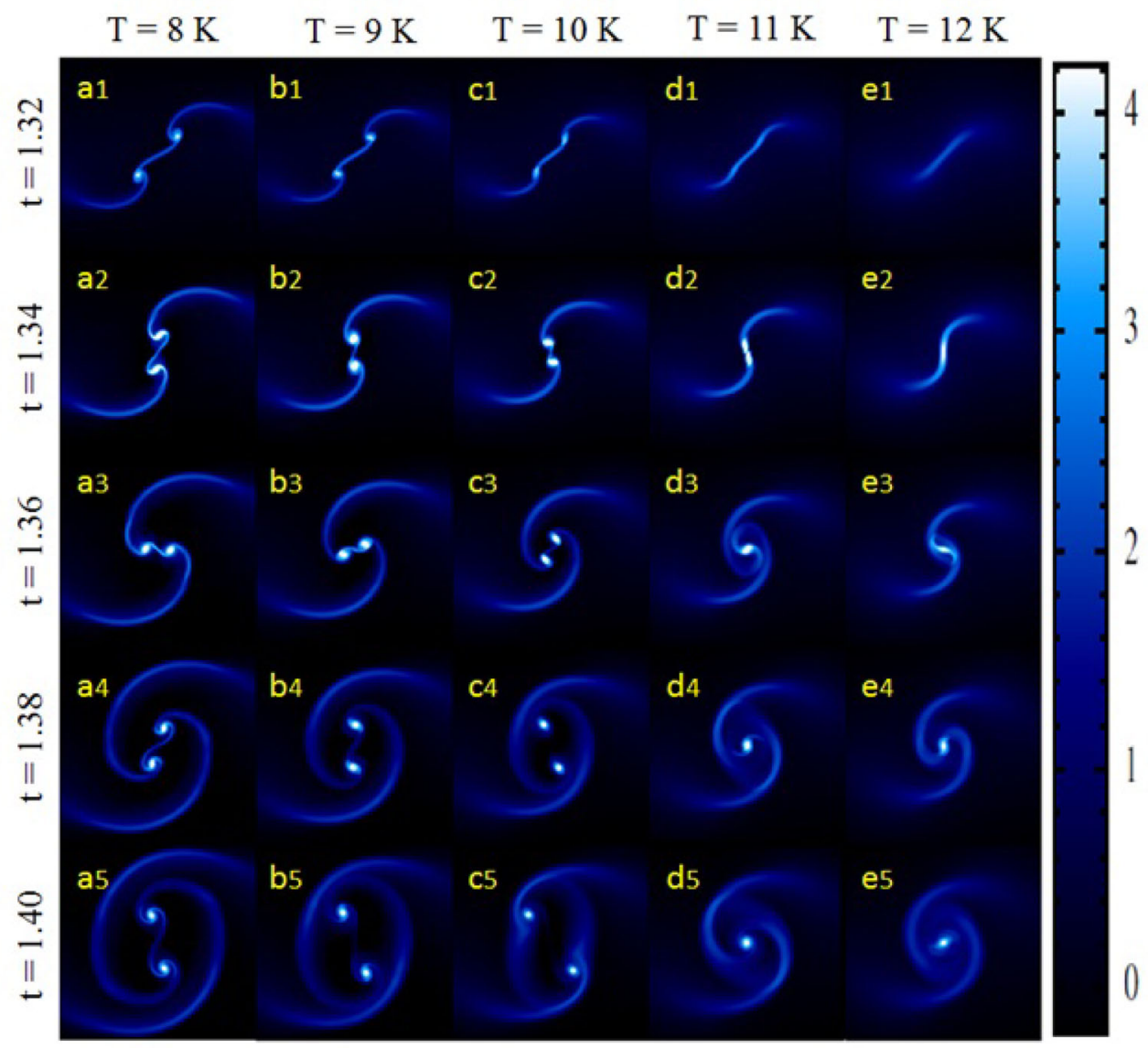

Figure 1. Simulation results for models A, B, C, D, and E. Each column of plots shows face-on views of the column density integrated along the z-axis for each particular model. The corresponding initial temperature is shown at the top of each column. The times corresponding to each row are given in units of $t_{\mathrm{ff}}$. The binary separations for each snapshot are, respectively, given by (a1) $d=313.184$ au, (a2) $d=224.943 \mathrm{au}$, (a3) $d=88.758 \mathrm{au},(\mathrm{a} 4) d=199.348$ au, (a5) $d=278.685 \mathrm{au},(\mathrm{b} 1) d=282.382 \mathrm{au}$, (b2) $d=200.013 \mathrm{au},(\mathrm{b} 3) d=151.047 \mathrm{au},(\mathrm{b} 4) d=251.859 \mathrm{au},(\mathrm{b} 5) d=343.235 \mathrm{au},(\mathrm{c} 1) d=262.515 \mathrm{au},(\mathrm{c} 2) d=$ $166.739 \mathrm{au},(\mathrm{c} 3) d=173.062 \mathrm{au},(\mathrm{c} 4) d=266.667 \mathrm{au},(\mathrm{c} 5) d=378.364 \mathrm{au}$. The horizontal and vertical dimensions of each plot in the $x y$-plane are $0.12 \times 0.12$ in dimensionless units. The colour bar on the right shows $\log (\Sigma)$ in dimensionless units. Each calculation was performed with 250025 SPH particles.

understanding of the physical processes involved in star formation (Duffin \& Pudritz 2008; Hubber et al. 2011; Loughnane, Redman \& Keto 2012; Frank et al. 2013). Among the recent efforts, we mention numerical models of the collapse of molecular clouds to protostellar densities from an initial Gaussian and Plummer density distribution (Gomez-Ramirez et al. 2012). The impact of magnetic fields on protostellar collapse and fragmentation has also been investigated in detail (for example Boss 2001; Peters et al. 2007; Donati et al. 2009; Bürzle et al. 2011).

Based on the results of his collapse calculations including radiative transfer in the smoothed particle hydrodynamics (SPH) framework, Stamatellos (2007) found that for densities below 




Figure 2. Same as in Fig. 1, but here we show edge-on views of the column densities integrated along the direction orthogonal to the $z$-axis. The horizontal and vertical dimensions in the $x z$-plane in each panel are $0.1 \times 0.05$ in dimensionless units, respectively.

Table 2. Summary of the maximum evolution time, as well as the maximum density and the maximum temperature obtained from the evolution of the models.

\begin{tabular}{lccccc}
\hline \multicolumn{1}{c}{ Model } & $\mathrm{A}$ & $\mathrm{B}$ & $\mathrm{C}$ & $\mathrm{D}$ & \\
\hline$t_{\max }(\mathrm{yr})$ & 47913.746 & 47913.746 & 47913.746 & 47913.746 & \\
$\rho_{\max }\left(\mathrm{g} \mathrm{cm}^{-3}\right)$ & $4.767 \times 10^{-11}$ & $2.380 \times 10^{-11}$ & $1.883 \times 10^{-11}$ & $7.551 \times 10^{-12}$ & \\
$T_{\max }(\mathrm{K})$ & 1298 & 922.5 & 878.4 & 530.2 & \\
Model & $\mathrm{E}$ & $\mathrm{F}$ & $\mathrm{G}$ & $\mathrm{H}$ & $\mathrm{I}$ \\
\hline$t_{\max }(\mathrm{yr})$ & 47913.746 & 47913.746 & 41443.078 & 47913.746 & 47913.746 \\
$\rho_{\max }\left(\mathrm{g} \mathrm{cm}^{-3}\right)$ & $4.643 \times 10^{-12}$ & $8.118 \times 10^{-14}$ & $8.909 \times 10^{-10}$ & $7.787 \times 10^{-12}$ & $5.042 \times 10^{-12}$ \\
$T_{\max }(\mathrm{K})$ & 421.5 & 116.7 & 2456 & 540.965 & 444.7 \\
\hline & & & & &
\end{tabular}
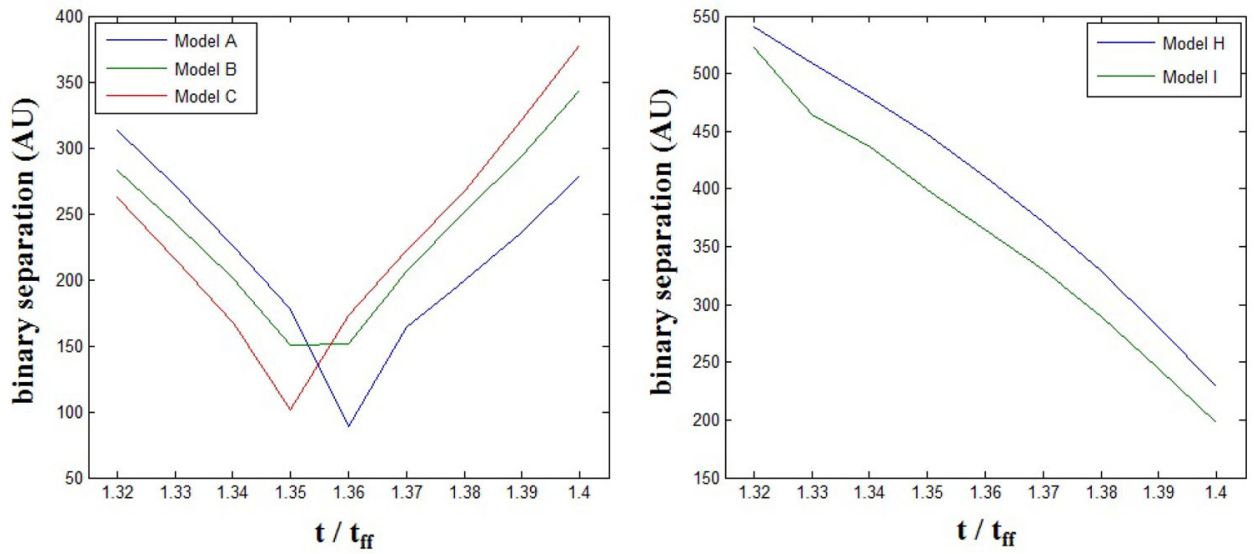

Figure 3. Evolution of the binary separation for models A, B, and C (left-hand panel) and for models H and I (right-hand panel) as a function of time up to $1.4 t_{\mathrm{ff}}$. The binary separation for the two protostars is determined by searching for the SPH particles with maximum density and their corresponding distance in simulation space.

$10^{-13} \mathrm{~g} \mathrm{~cm}^{-3}$, the temperature of the gas can be approximated by the following expression

$T=5 \mathrm{~K}\left(\frac{\rho}{10^{-13} \mathrm{~g} \mathrm{~cm}^{-3}}\right)^{0.08}$.
The above relation describes the slow heating process in collapsing cores within the density range $10^{-18} \mathrm{~g} \mathrm{~cm}^{-3}<\rho<10^{-13} \mathrm{~g} \mathrm{~cm}^{-3}$. Within this range the effective equation of state of the gas is almost isothermal. Beyond this density range the temperature creeps up much more rapidly due to a rise in the opacity of the gas. As a result, the model 


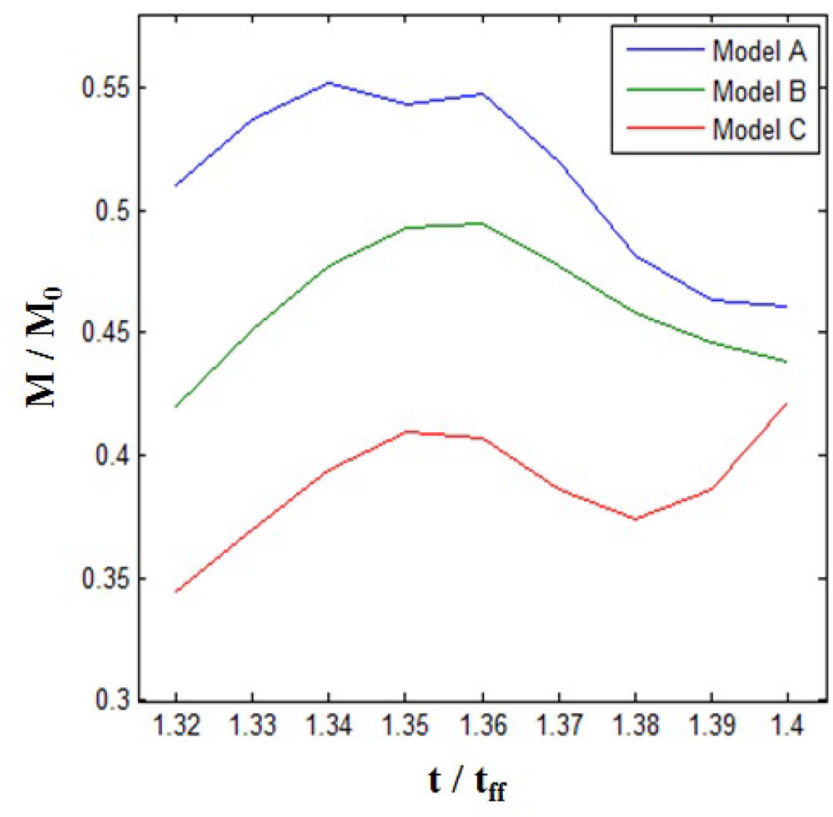

Figure 4. Evolution of the total relative mass fraction for the protostellar binaries in models $\mathrm{A}, \mathrm{B}$, and $\mathrm{C}$ as a function of time up to $1.4 t_{\mathrm{ff}}$.

(equation 1) cannot be used any more to estimate the temperature of the cloud.

Furthermore, in a recent paper, Launhardt et al. (2013) revealed the decisive role which is played by physical quantities such as the initial temperature and the initial density structure of the cloud in determining the final end products of gravitationally collapsing cores. Molecular gas with a slightly enhanced metallicity compared to the metallicity which is normally found in the solar neighbourhood could provide an efficient cooling mechanism in collapsing clouds. Metal-rich gas allows molecular cloud cores to remain isothermal for a longer period of time because a cloud with a relatively higher metallicity would be subject to more efficient radiative cooling, hence prolonging its isothermal state during collapse compared to clouds in which metal-poor conditions prevail (see for example Omukai 2000). To mimic the effect of varying the metallicity, we have used a barotropic equation of state and analysed the evolution of rotating collapsing molecular cloud cores by varying the value of the transition density at which the gas switches its thermodynamic behaviour from isothermal to adiabatic. A similar strategy has been adopted by Gomez-Ramirez et al. (2012). The main difference of their setup with our work is that we consider initial conditions with uniform density rather than Gaussian and Plummer models. On the other hand, observations of molecular cloud cores have revealed values for the lowest temperatures down to at least $8 \mathrm{~K}$ with typical values of $10 \mathrm{~K}$ (Benson \& Myers 1989; Ward-Thompson, Andr $\&$ Kirk 2002). Therefore, we explored a slightly unorthodox but yet possible range of initial temperatures in star-forming molecular clouds between 8 and $12 \mathrm{~K}$, keeping in mind that several physical mechanisms can cause prestellar gas to heat up or to cool down from the commonly adopted value of $10 \mathrm{~K}$. For example, cosmic rays can ionize the medium causing the temperature to climb up from $10 \mathrm{~K}$ to a certain limit or line radiation from molecules can cool down the gas below this value (Wiener, Zweibel \& Oh 2007; Indriolo 2011; Padovani, Hennebelle \& Galli 2013). This leads us to consider the possible role of the initial thermal state of star-forming clouds in determining the properties of binary protostar systems. We focus on the relation between the binary separation of the protostars formed through gravitational collapse and the initial temperature of the cloud, as well as the possible emergence of protostellar objects as a result of secondary fragmentation.

The numerical models of cloud collapse recently reported in Gomez-Ramirez et al. (2012) demonstrate that there exists a tendency for models with initial Plummer and Gaussian density distributions to switch from the isothermal to the adiabatic regime at slightly lower critical densities. Models with a lower critical density show enhanced fragmentation compared to models with higher critical densities. Keeping this in mind, we also examine the relation between the critical density and the number of fragments resulting from the gravitational collapse. We also present an analysis of the extreme values of density and temperature within the fragments. The structure of the paper is as follows. In Section 2, we provide details on the SPH code GRADSPH which has been used in this work. We describe the setup of the numerical models in Section 3. Section 4 gives an overview of the results of our simulations. Finally, our conclusions are presented in Section 5.
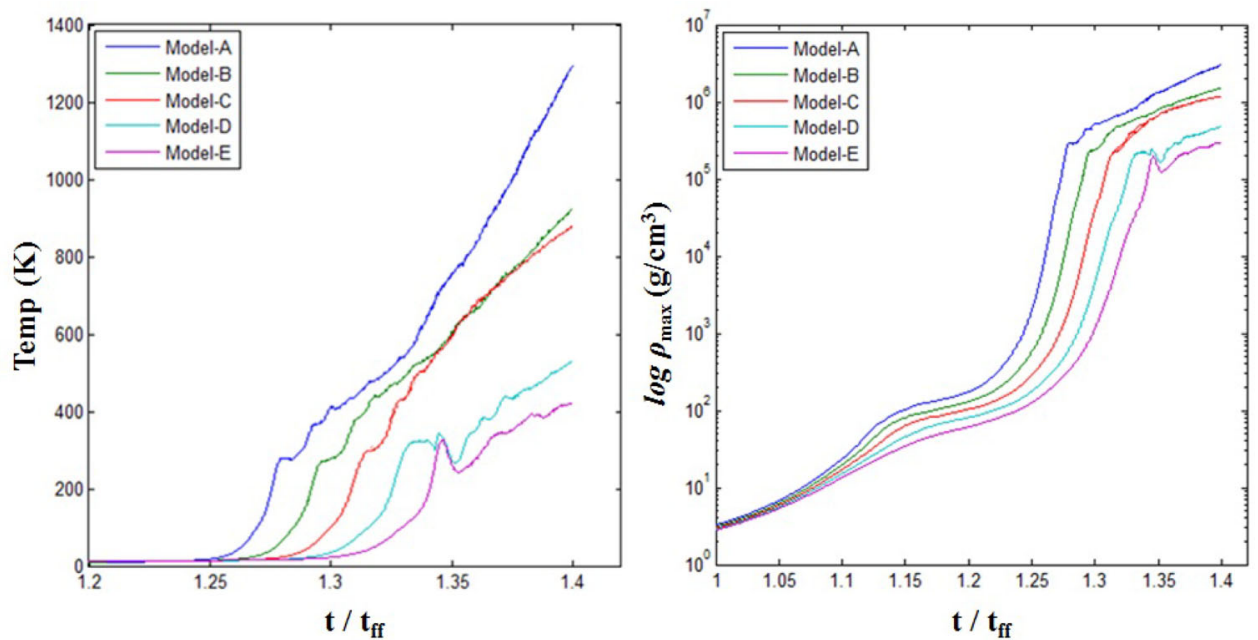

Figure 5. Time evolution of the maximum temperature within the collapsing cloud for models A, B, C, D, E (left-hand panel), and the time evolution of the maximum density for the same set of models (right-hand panel). 

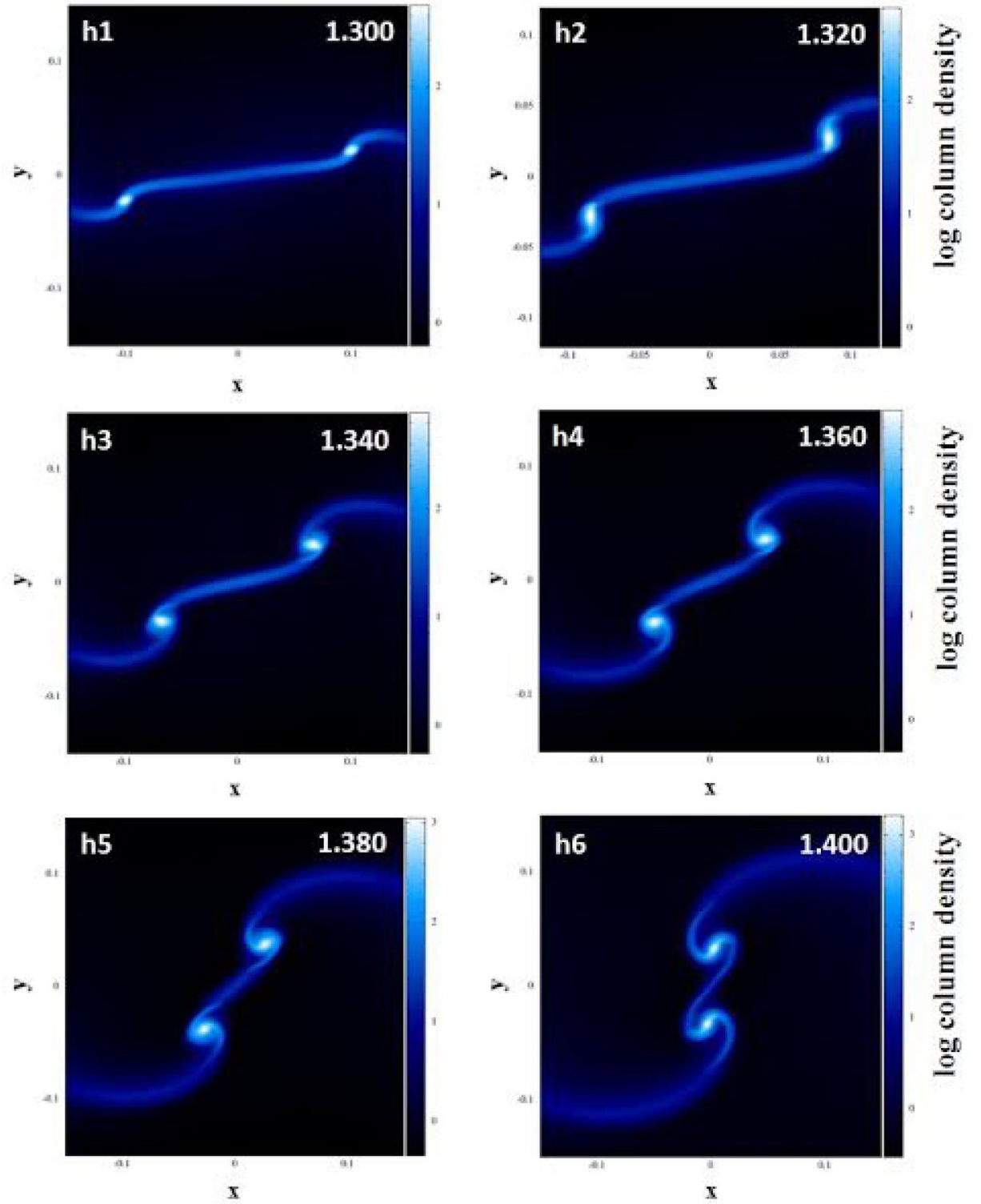

Figure 6. Simulation results for model $\mathrm{H}$. Each plot shows a face-on view the column density integrated along the $z$-axis. The times corresponding to each row are given in units of $t_{\mathrm{ff}}$. The binary separations for each snapshot are, respectively, given by (h1) $d=590.130 \mathrm{au}$, (h2) $d=540.667 \mathrm{au}$, (h3) $d=479.196$ au, (h4) $d=410.897 \mathrm{au},(\mathrm{h} 5) d=328.628 \mathrm{au},(\mathrm{h} 6)=228.438 \mathrm{au}$. The horizontal and vertical dimensions of each plot in the $x y$-plane are $0.15 \times 0.15$ in dimensionless units. The colour bar on the right shows $\log (\Sigma)$ in dimensionless units. Each calculation was performed with $250025 \mathrm{SPH}$ particles.

\section{NUMERICAL METHOD}

For the hydrodynamical models of molecular could collapse considered in this work, we use GRADSPH, a tree-based, parallel particlebased hydrodynamics code based on the SPH method written in FORTRAN 90 (Vanaverbeke et al. 2009). The code has several features which enable us to model gravitational cloud collapse, including the treatment of gravitational forces using a tree-based gravity (TCG) method combined with a variable gravitational softening length formalism (for details see Price \& Monaghan 2007; Vanaverbeke et al. 2009). The SPH formalism implemented in GRADSPH is based on deriving the SPH equations from a variational principle (Price 2012). The reliability of the code has been established through an extensive series of tests on standard benchmark problems (Vanaverbeke et al. 2009) and the code has recently been extended to MHD (Vanaverbeke, Keppens \& Poedts 2013).
According to Price (2012, see also Gingold \& Monaghan 1977), the basic equations of motion used for evolving the SPH particles in GRADSPH are given as follows:

$$
\frac{\mathrm{d} \boldsymbol{v}_{i}}{\mathrm{~d} t}=-\sum_{j=1}^{N} m_{j}\left[\frac{P_{i}}{\rho_{i}^{2} \Omega_{i}} \nabla_{i} W\left(r_{i j}, h_{i}\right)+\frac{P_{j}}{\rho_{j}^{2} \Omega_{j}} \nabla_{i} W\left(r_{i j}, h_{j}\right)\right],
$$

in which the coefficients $\Omega_{i}$ are defined as

$\Omega_{i}=1-\frac{\partial h_{i}}{\partial \rho_{i}} \sum_{j=1}^{N} m_{j} \frac{\partial W\left(r_{i j}, h_{j}\right)}{\partial h_{j}}$.

In the above equations, $N$ is the total number of particles in the simulation, $P_{i}$ and $\rho_{i}$ denote the density and pressure of a particle labelled $i, h_{i}$ is its smoothing length and $m_{i}$ denotes its mass. The position and velocity vectors for the particles are denoted by $\boldsymbol{r}_{i}$ 


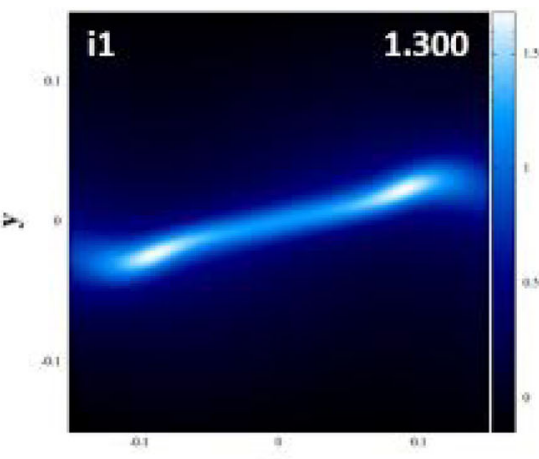

X
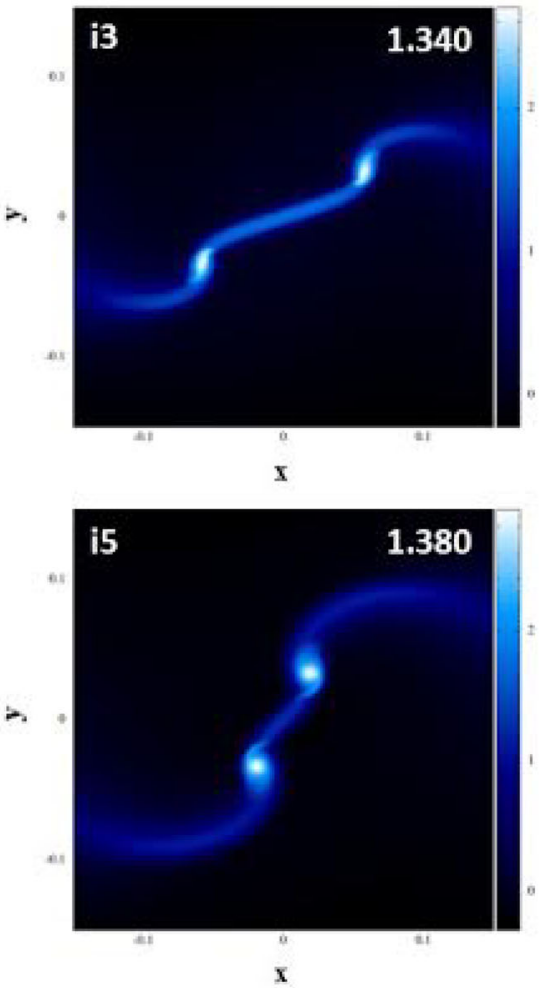

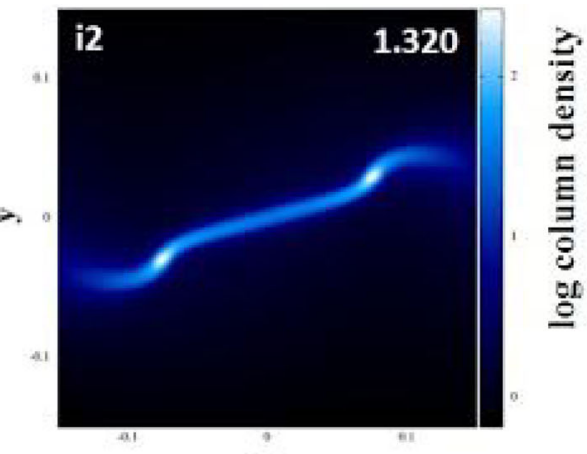

x.
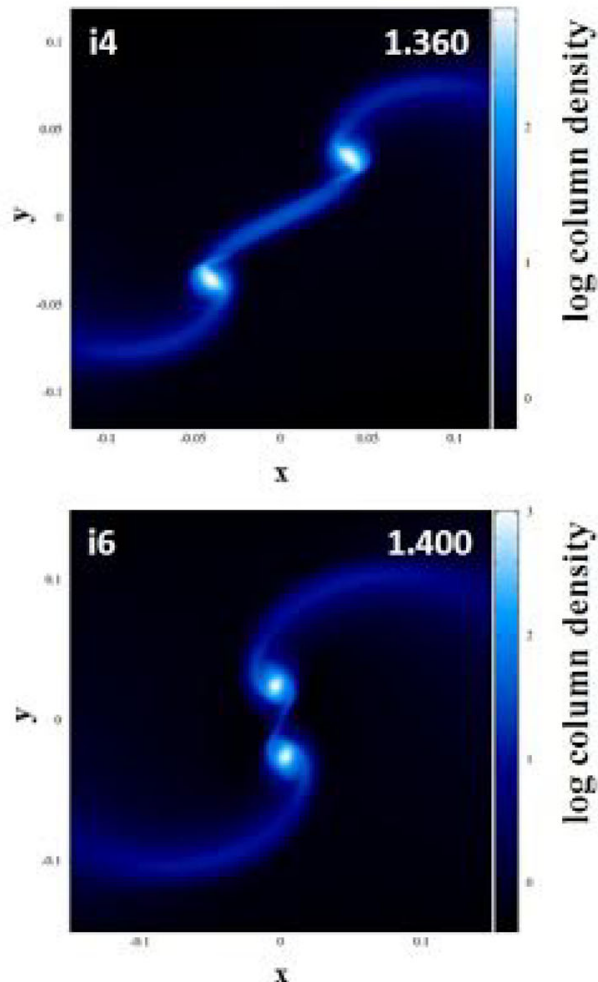

Figure 7. Simulation results for model I. Each plot shows a face-on view the column density integrated along the $z$-axis. The times corresponding to each row are given in units of $t_{\mathrm{ff}}$. The binary separations for each snapshot are, respectively, given by (i1) $d=531.065 \mathrm{au},(\mathrm{i} 2) d=522.757 \mathrm{au}$, (i3) $d=435.9223$ au, (i4) $d=364.861 \mathrm{au}$, (i5) $d=289.241 \mathrm{au}$, (i6) $=197.608$ au. The horizontal and vertical dimensions of each plot in the $x y$-plane are $0.15 \times 0.15$ in dimensionless units. The colour bar on the right shows $\log (\Sigma)$ in dimensionless units. Each calculation was performed with 250025 SPH particles.

and $\boldsymbol{v}_{i}$, respectively, and $\boldsymbol{r}_{i j}=\boldsymbol{r}_{i}-\boldsymbol{r}_{j}$. The coefficients $\Omega_{i}$ selfconsistently take into account the effect of a variable smoothing length (Price 2012). In this paper, we close the system of equations by specifying a barotropic equation of state $P=P(\rho)$ for the gas which will be specified in Section 3 . In this way, we eliminate the need to solve an energy equation. The code uses time-dependent artificial viscosity terms in order to capture shock waves and prevent particle penetration. In order to maintain hydrodynamic stability, we use the signal-velocity approach introduced by Price and Monaghan (Price \& Monaghan 2007) to calculate the artificial viscosity terms and the Courant time step. The Courant number is set to 0.1 in our simulations. This choice is found to be sufficient to maintain stability in our calculations. In the artificial viscosity terms, we set the parameters $C_{a}$ and $\alpha_{\min }$ in equations 34 and 35 in Vanaverbeke et al. (2009) to 0.2 and 0.5 , respectively.
The particle densities are computed from the standard SPH summation equation (Vanaverbeke et al. 2009; Price 2012) by summing the contribution from all the particles which overlap with the position of particle $i$, using a weighting function $W\left(\boldsymbol{r}_{i}-\boldsymbol{r}_{j}, h_{i}\right)$ :

$\rho_{i}=\sum_{j=1}^{N} m_{j} W\left(\boldsymbol{r}_{i}-\boldsymbol{r}_{j}, h_{i}\right)$.

In the above expression, $W\left(\boldsymbol{r}_{i}-\boldsymbol{r}_{j}, h_{i}\right)$ is a smooth differentiable function, referred to as the smoothing kernel or the interpolating kernel. GRADSPH uses the standard M4-kernel or cubic spline kernel (Price 2012). The smoothing length is updated at each time step by 



Figure 8. Time evolution of the maximum temperature within the collapsing cloud for models $\mathrm{H}$ and I (left-hand panel), and the time evolution of the maximum density for the same set of models (right-hand panel).
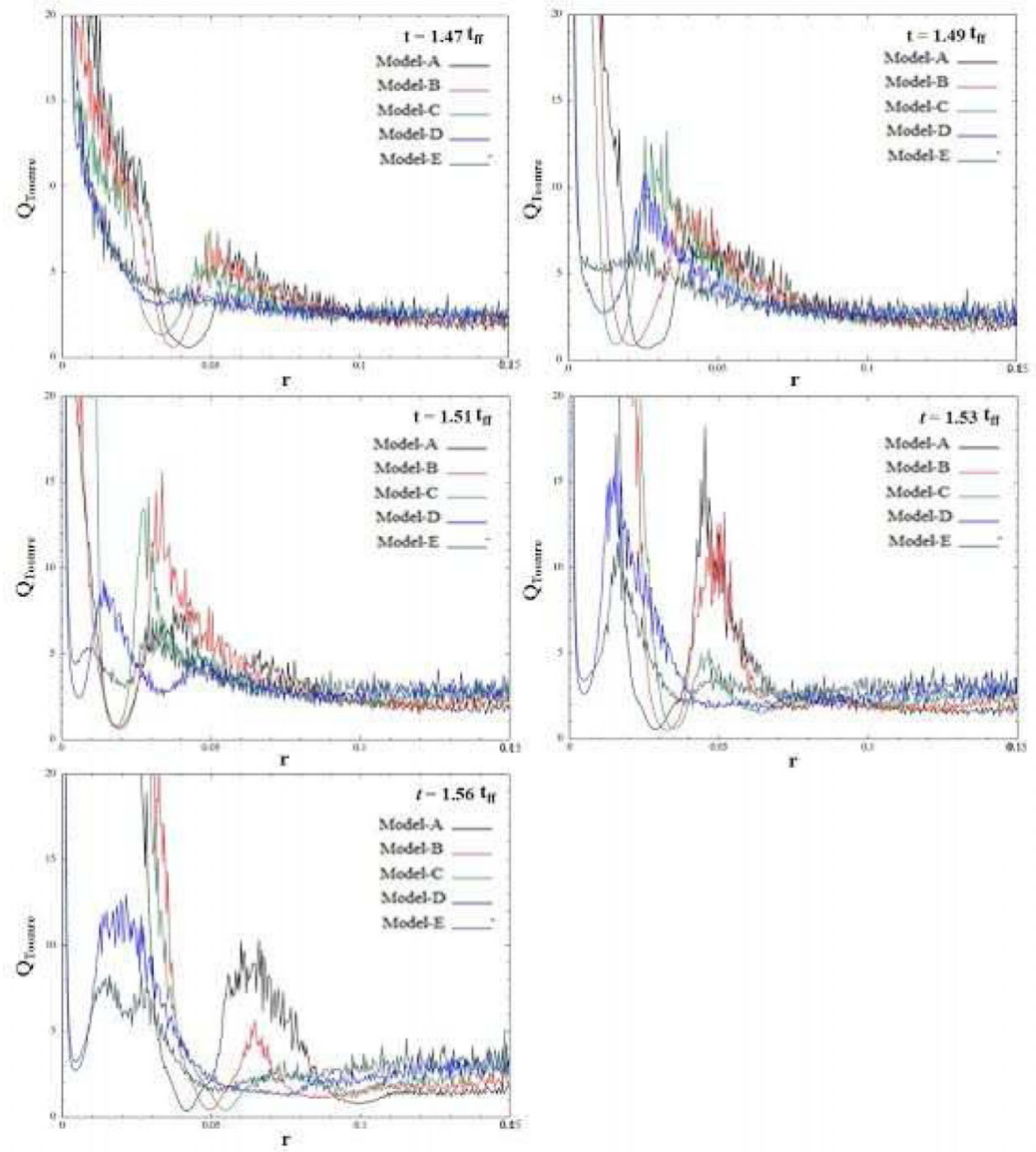

Figure 9. Traces of the azimuthal average of the Toomre parameter values as a function of the radial distance $r$ in the cloud mid-plane for models A-E at times $t=1.47,1.49,1.51,1.53$, and $1.56 t_{\mathrm{ff}}$, respectively.

iteratively solving the following equation for each particle (Price 2012)

$\frac{4 \pi}{3}\left(2 h_{i}\right)^{3} \rho_{i}=m_{i} N_{\mathrm{opt}}$

in which $N_{\text {opt }}$ is the number of neighbours contained within the smoothing sphere of each particle. $N_{\text {opt }}$ is set to 50 in our calculations. In this way, we ensure that a constant mass is contained within the smoothing sphere for every SPH particle so that the code adapts its resolution to keep track of density changes that may occur during the dynamical evolution of the fluid. The system of ordinary differential equation (2), which updates the positions and velocities of the particles, is solved using a predictor-corrector scheme combined with an individual particle time stepping method.

As mentioned before, the self-gravity of the gas is treated using the TCG method. We compute the gravitational acceleration of 




Figure 10. Snapshots of column density integrated along the rotational axis during the evolution of model G. The horizontal and vertical dimensions in the $x y$-plane in each panel are $0.15 \times 0.15$ in dimensionless units. The colour bar shows $\log (\Sigma)$ in dimensionless units. The times in each row are in units of $t_{\mathrm{ff}}$.

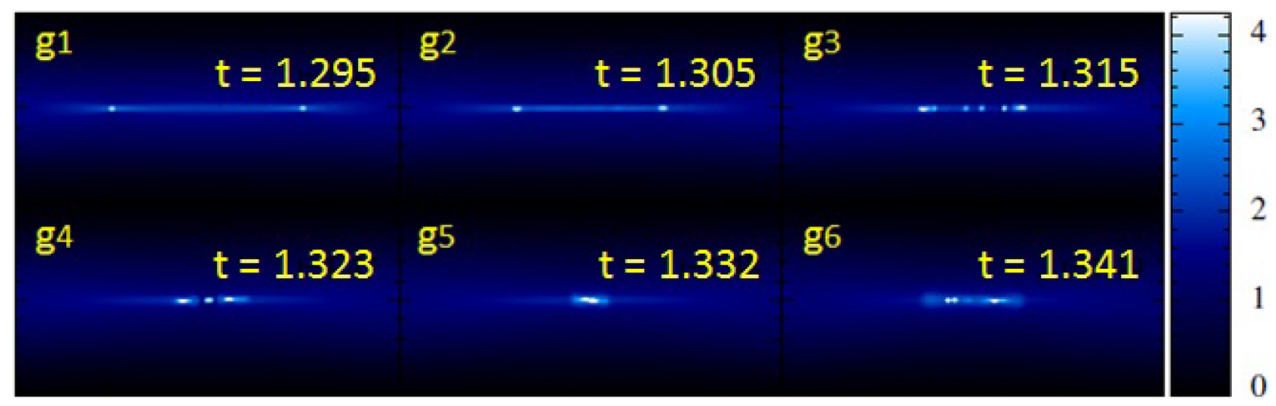

Figure 11. Same as in Fig. 10, but here we show the column densities integrated along the direction orthogonal to the rotational axis. The horizontal and vertical dimensions in the $x z$-plane in each panel are $0.1 \times 0.05$ in dimensionless units.

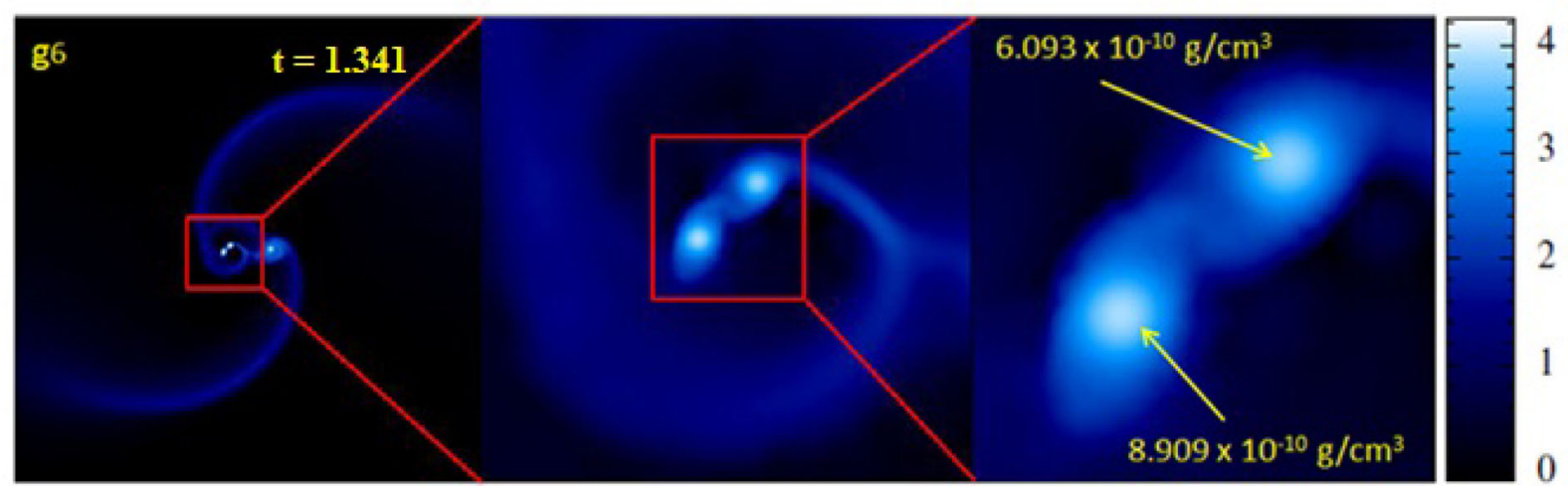

Figure 12. Detailed snapshots of the column density integrated along the $z$-axis during the evolution of model G. The first panel on the left has dimensions $0.25 \times 0.25$, while the other two panels are successive enlargements of the first one. The colour bar on the right shows $\log (\Sigma)$ in dimensionless units. The time indicated in each snapshot is given in units of $t_{\mathrm{ff}}$. 


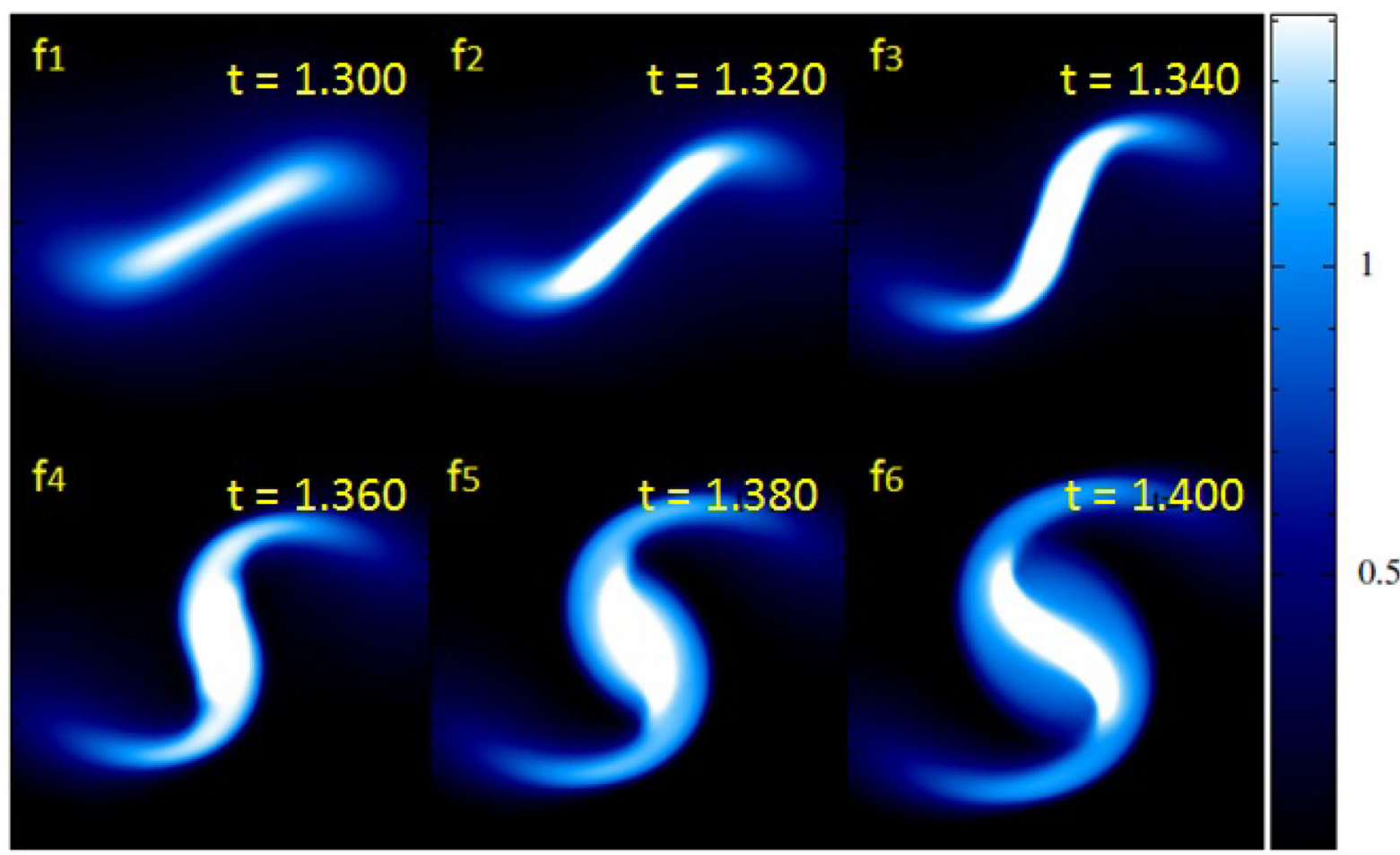

Figure 13. Snapshots of column density integrated along the rotational axis during the evolution of model $\mathrm{F}$. The horizontal and vertical dimensions in the $x y$-plane in each panel are $0.15 \times 0.15$ in dimensionless units. The colour bar shows $\log (\Sigma)$ in dimensionless units. The times in each row are in units of $t_{\mathrm{ff}}$. Each calculation was performed with $250025 \mathrm{SPH}$ particles.

each particle using a Barnes-Hut tree algorithm. The opening angle for the tree is an important parameter which we set to $\theta=0.7$. The same Barnes-Hut tree algorithm is also used to update the list of neighbours of the SPH particles. We use the cubic spline kernel to soften gravitational forces and include the correction terms derived by Price $\&$ Monaghan (2007) to ensure the conservation of energy when dealing with variable particle smoothing lengths. The gravitational acceleration of particle $i$ is thus given by

$$
\begin{aligned}
\boldsymbol{g}_{i}= & -G \sum_{j=1}^{N}\left[\Phi^{\prime}\left(r_{i j}, h_{i}\right)+\Phi^{\prime}\left(r_{i j}, h_{j}\right)\right] \boldsymbol{e}_{i j} \\
& -\frac{G}{2} \sum_{j=1}^{N}\left[\frac{\zeta_{i}}{\Omega_{i}} \nabla_{i} W\left(r_{i j}, h_{i}\right)+\frac{\zeta_{j}}{\Omega_{j}} \nabla_{i} W\left(r_{i j}, h_{i}\right)\right]
\end{aligned}
$$

in which the quantities $\zeta_{i}$ are defined as

$\zeta_{i}=\frac{\partial h_{i}}{\partial \rho_{i}} \sum_{j=1}^{N} m_{j} \frac{\partial \Phi\left(r_{i j}, h_{i}\right)}{\partial h_{i}}$,

$\Phi(r, h)$ is the softened gravitational potential of a particle, and $\frac{\partial \Phi(r, h)}{\partial h}$ is the derivative of the potential with respect to the smoothing length. Expressions for the softened potential and its derivatives are tabulated in Price \& Monaghan (2007).

\section{INITIAL CONDITIONS}

The initial conditions for the cloud collapse calculations considered in this a paper are variants of the Boss and Bodenheimer collapse test with initial conditions described in Burkert \& Bodenheimer (1993). We used this test previously to validate the GRADSPH code on collapse calculations (see section 8.2.3 in Vanaverbeke et al. 2009). The initial condition that we take as our standard model is a solar mass cloud with uniform density and radius $R=5 \times 10^{16}$ $\mathrm{cm}$. The cloud is assumed to be in solid body rotation around the $z$-axis of the coordinate system with an angular velocity equal to $\omega=7.2 \times 10^{-13} \mathrm{rad} \mathrm{s}^{-1}$ and is rotating counterclockwise. The mean initial density of the cloud is $\rho_{0}=3.8 \times 10^{-18} \mathrm{~g} \mathrm{~cm}^{-3}$. The chemical composition of the cloud is assumed to be a mixture of hydrogen and helium gas with mean molecular weight $\mu=3$. The initial condition is characterized by the parameters $\alpha$ and $\beta$, which correspond to the ratio of thermal and kinetic energy with respect to the gravitational potential energy of the cloud. These parameters are defined as follows:

$\alpha=\frac{5 R k T}{2 G M \mu m_{\mathrm{h}}}$,

$\beta=\frac{R^{3} \omega^{2}}{3 G M}$,

where $G$ is the gravitational constant, $k$ is the Boltzmann constant, and $m_{\mathrm{h}}$ denotes the mass of the hydrogen atom. For the standard initial temperature of $10 \mathrm{~K}$, the initial values for $\alpha$ and $\beta$ are 0.26 and 0.16 , respectively. In our models, the value of $\alpha$ changes by modifying the initial temperature, whereas $\beta$ will be kept fixed at its standard value. The mean free-fall time of the initial condition is given by

$t_{\mathrm{ff}}=\sqrt{\frac{3 \pi}{32 G \rho_{0}}}$

and is $33968 \mathrm{yr}$ for the standard initial condition defined above. The initial setup is implemented in our SPH code by placing equal-mass particles on a hexagonal closely packed lattice and retaining only the particles within the initial cloud radius. The code uses internal dimensionless units which are defined by setting $G=M=R=1$. 


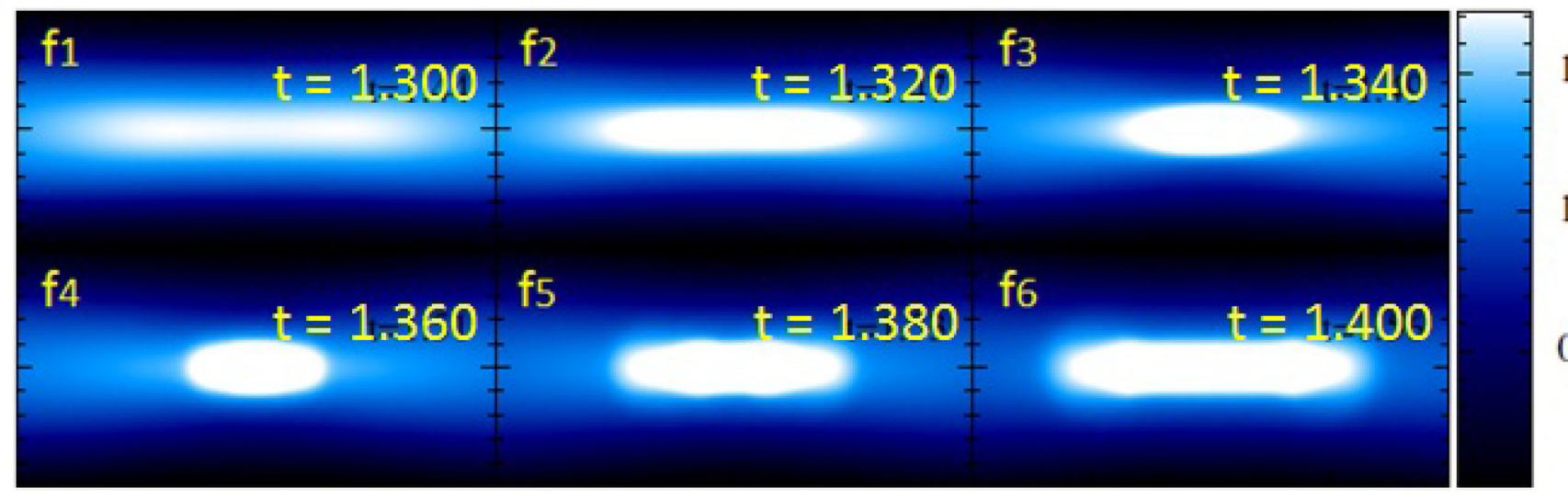

Figure 14. Same as in Fig. 13, but here we show the column densities integrated along the direction orthogonal to the rotational axis. The horizontal and vertical dimensions in the $x z$-plane in each panel are $0.1 \times 0.05$ in dimensionless units.

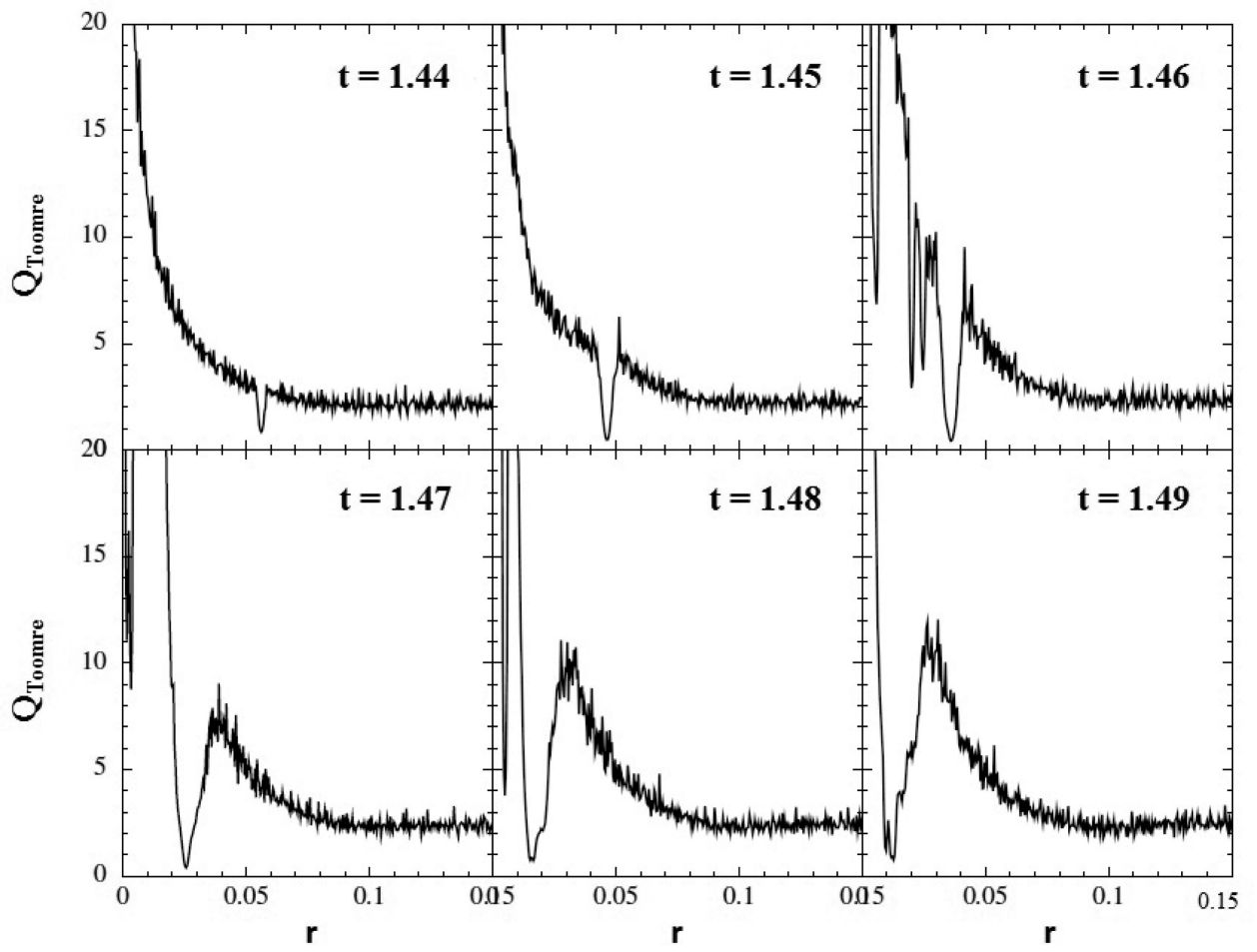

Figure 15. Traces of the Toomre parameter against radial distance $r$ measured in the equatorial plane for model $\mathrm{F}$. The times for each snapshot are given in units of $t_{\mathrm{ff}}$.

To initiate fragmentation of the cloud models, we add an azimuthal density perturbation to the uniform initial condition with a certain mode number $m$ and amplitude $A$ which has the following form:

$\rho=\rho_{0}(1+A \sin (m \phi)]$,

where $\phi$ is the azimuthal angle in spherical coordinates $(r, \phi, z)$. We implement this perturbation by perturbing the azimuthal angle of the SPH particles to a new angle $\phi *$ which is determined by solving the equation

$\phi=\phi^{*}+\frac{A \sin \left(m \phi^{*}\right)}{m}$.

A common mode number $m=2$ is adopted for all the models listed in Table 1 while azimuthal density perturbations of amplitude $A=$ 0.1 and 0.25 are considered for models $(\mathrm{A}, \mathrm{B}, \mathrm{C}, \mathrm{D}, \mathrm{E}, \mathrm{F}, \mathrm{G})$ and models (H and I), respectively. The thermodynamic behaviour of the gas during cloud collapse is approximated by adopting a barotropic equation of the state as suggested by Tohline (1982) and Matsunaga $\&$ Inutsuka (2004). The pressure and sound velocity of the gas are given by the expressions

$P=\rho c_{0}^{2}\left[1+\left(\frac{\rho}{\rho_{\text {crit }}}\right)^{\gamma-1}\right]$,

and

$c_{s}=c_{0}\left[1+\left(\frac{\rho}{\rho_{\text {crit }}}\right)^{\gamma-1}\right]^{1 / 2}$,

where $c_{0}$ is the initial sound velocity, $\rho_{\text {crit }}$ is the critical density which determines the transition point from isothermal to adiabatic 




Figure 16. Traces of the surface density against radial distance $r$ measured in the equatorial plane for model F. The times for each snapshot are given in units of $t_{\mathrm{ff}}$.

behaviour of the gas, and $\gamma$ is the adiabatic exponent which we set to $5 / 3$. Our standard value for the critical density is $\rho_{\text {crit }}=$ $5 \times 10^{-14} \mathrm{~g} \mathrm{~cm}^{-3}$. The initial models are allowed to evolve under the action of the short-range hydrodynamical forces and the selfgravity of the cloud. No external radiation feedback is taken into consideration during evolution of our models.

In order to avoid artificial fragmentation in our models because of insufficient resolution, we need to satisfy the resolution criterion derived by Bate \& Burkert (1997) in our models. This is achieved by keeping the minimum resolvable mass of the code, which equals $M_{\text {min }}=2 N_{\text {opt }} m$, below the local Jeans mass. Since the Jeans mass decreases during the isothermal collapse stage and increasing again when the gas becomes optically thick and adiabatic, this condition defines an upper limit to the mass of an SPH particle following (Arreaga-Garcia et al. 2007; Vanaverbeke et al. 2009):

$$
m<\frac{\pi^{3 / 2} c_{0}^{3}}{2 N_{n, \text { opt }} \rho_{\text {crit }}^{1 / 2} G^{3 / 2}} .
$$

The present numerical study is conducted by taking a total number of SPH particles equal to $N=250025$ in the initial condition and setting the number of neighbours to $N_{\text {opt }}=50$. Table 1 provides an overview of the models which have been used to examine the evolution of the binary separation as a function of the initial temperature of the clouds as well as the impact of the changes in the critical density along with changes in perturbation amplitude. The table gives, for the nine models labelled A-I, the values of the initial temperature, the sound velocity, the critical density, the resulting binary separation when applicable, as well as the final outcome of the simulations which will be discussed in Section 4. It can be seen that models A-E explore the effect of changes in the temperature with the critical density kept at its standard value. In models F and G, on the other hand, the initial temperature is set to $10 \mathrm{~K}$ and $\rho_{\text {crit }}$ takes on different values to examine the effect of this parameter on the evolution of the cloud models. The last two models labelled $\mathrm{H}$ and I reveal the effects of strength of perturbation amplitude on evolution of the collapsing cloud.

In our models, fragmentation proceeds in two stages. In the first stage, a rotating disc like structure forms in the centre of the cloud as a result of the conservation of angular momentum. In the second stage, this disc fragments into protostellar fragments once the gas has become optically thick and evolves adiabatically because of non-axisymmetric gravitational instabilities. The growth of this kind of gravitational instabilities is governed by the Toomre parameter for a disc with surface density $\Sigma$ and epicyclic frequency $\kappa$ (Toomre 1964). This parameter is defined as

$Q=\frac{c_{s} \kappa}{\pi G \Sigma}$,

and the value of $Q$ must be smaller than unity to enable nonaxisymmetric instabilities to grow within the disc. Plots of the radial behaviour of the Toomre parameter at various stages of the collapse will be discussed in Section 4. For visualization of the results of our simulations, we use the visualization tool SPLASH developed and made publicly available to the community by Daniel Price (Price 2007).

\section{RESULTS AND DISCUSSION}

Fig. 1 shows successive column density maps of the evolution of models A-E at five different times indicated to the left of the columns. The initial temperature of the models is indicated at the top of each column. The column density maps are top down views 
in the $x y$-plane where the density distribution has been integrated along the rotational axis of the cloud. Fig. 2 shows the corresponding edge-on column density maps in the $x z$-plane orthogonal to the rotational axis. Every single square panel in Fig. 1 has a physical dimension of approximately $401 \times 401$ au. In Fig. 2 every single panel has physical dimensions of approximately $401 \times 167 \mathrm{au}$, respectively. The outcome of each simulation model and the separation of the binary at the end of each run are summarized in Table 1. Table 2 contains the maximum time, $t_{\max }$, expressed in years, during which we have been able to evolve the model before the timestep became prohibitively small, as well as the maximum density $\rho_{\max }$ and the maximum temperature $T_{\max }$ attained during the evolution of each model. The rise in initial temperature going from model A to E has a drastic impact on the evolution of the cloud collapse. Models with initial temperatures up to standard value of $10 \mathrm{~K}$ develop binary systems, whereas molecular cores with temperatures above $10 \mathrm{~K}$ fail to develop binaries yielding only single protostars instead. Changing the initial temperature also has a big impact on the separation of the binaries in the range $8<T<=10 \mathrm{~K}$. For example, raising the temperature from 8 to $9 \mathrm{~K}$ leads to change in the final binary separation of around $\Delta d=64$ au, while a further increase from 9 to $10 \mathrm{~K}$ leads to a change around $\Delta d=35 \mathrm{au}$. The panels in Fig. 2 also clearly show the formation of the intermediate disc structure which fragments into protostars for $T<=10 \mathrm{~K}$.

The phase in which the collapsing fragments are still connected by a bar-like structure is comparatively longer in molecular cloud cores with temperatures below the conventional value of $10 \mathrm{~K}$. This is illustrated in Fig. 1 by the snapshots (a5, b5, and c5). It can be seen that this connecting filament starts to disappear earlier in model $\mathrm{C}$ than in models $\mathrm{A}$ and $\mathrm{B}$. There is also an important difference in evolution of the orbital elements of the models with increasing temperature for models $\mathrm{A}, \mathrm{B}$, and $\mathrm{C}$ which produce binary systems as the final products. The left-hand panel of Fig. 3 shows the evolution of the binary separation with time for these models. The time is indicated in units of the initial freefall time of the cloud. Each model shows a qualitatively similar evolution showing a minimum in the binary separation, after which the separation increases up to the end of the simulation. The physical reason for this behaviour is likely connected to the accretion of high angular momentum gas by the protostars from the surrounding envelope. However, there are important differences in terms of the speed of this evolution and the minimum separation of the binaries. Colder molecular cloud evolves slower than the hotter ones in the sense that the minimum separation is attained later in the course of the evolution. Note that at the end of the evolution, the faster evolving hotter cores have reached larger binary separations than the colder ones.

We approximately estimate the masses of the evolving fragments by locating the particle with the highest density $\rho_{\max }$ within each fragment and include all particles whose density is higher than $f \rho_{\max }$, where $f$ is set to 0.001 . The velocity and position of each fragment is determined from the position and velocity of the centre of mass of the clump, respectively. A similar procedure has been adopted by Arreaga-Garcia et al. (2007). Finally, the orbital elements of the binaries are determined from the positions, velocities, and masses of the fragments.

Fig. 3 shows that the binary separation in models (A, B, C) mainly follows the variations in semi-major axis and is less affected by the evolution of the orbital eccentricities which remain high $(\sim 0.5-0.6)$ throughout of the evolution. Fig. 4, on the other hand, shows that the initial thermal state of the clouds in decisive in determining the final mass fraction included in the binary system. The cooler the core, the more mass is involved in the binary fragmentation. The coolest model A has more than 50 per cent of its mass included at $t=1.35 t_{\mathrm{ff}}$. Since about half of the mass of the cloud is at most included in the binaries at the end of our simulations, we expect that the orbital elements of the binaries reported after $1.4 t_{\mathrm{ff}}$ are unlikely to represent the final orbital elements but are rather indicative of the trend of their further evolution. In particular, we expect that the increasing trend of the binary separation will continue despite the fact that we were unable to prolong the simulations for much longer than $1.4 t_{\mathrm{ff}}$. We also note that the decline of the mass fraction which can be seen in Fig. 4, but is only temporary for model $\mathrm{C}$ because this model evolves faster, is likely caused by mass exchange between the fragments and their surrounding disc like structure which could be the result of the tidal forces exerted by the companions. The symmetry of the initial conditions $(m=2)$ implies that the binary mass ratio is always close to unity.

Fig. 5 shows the time evolution of the maximum temperature for models A-E (left-hand panel) as well as the time evolution of the maximum of the density in the right-hand panel. Comparison of the models shows that for colder molecular cloud cores, the clouds are subject to an earlier increase in temperature along with a faster transition to the adiabatic regime. It is also evident that the final temperature of the models is highly dependent on their initial thermal state with colder models reaching higher final temperatures. The logarithmic density profiles shown in the right-hand panel indicate a similar trend for the maximum density. Here, the colder models reach higher final densities because self-gravitating fragments which have reached the adiabatic heating phase are formed earlier during the course of their evolution.

Figs 6-8, respectively, show top down views of the evolution of models $\mathrm{H}$ and $\mathrm{I}$ as well as the evolution of their maximum density and temperature, respectively. In these models, the temperature is raised above the nominal value of $10 \mathrm{~K}(11 \mathrm{~K}$ for model $\mathrm{H}$ and $12 \mathrm{~K}$ for model I) and a bigger perturbation amplitude is applied $(A=$ 25 per cent). Because of the increased strength of the perturbation, models $\mathrm{H}$ and $\mathrm{I}$ form binary systems at the end of the simulation, and thus clearly indicate that the fragmentation process is a function of both the thermal energy of the core and the strength of the initial perturbation.

Comparison of the results of models A-C and $\mathrm{H}$ and I leads to several conclusions. First, cold molecular cores $\left(T_{0}=8,9,10 \mathrm{~K}\right)$ with a smaller amplitude of the initial azimuthal density perturbation $(A$ $=10$ per cent) give birth to protobinary systems that evolve relatively quickly and include protostellar fragments that reach higher densities and temperatures compared to those in models $\mathrm{H}$ and $\mathrm{I}$. The binaries in models $\mathrm{A}-\mathrm{C}$ attain minimum separations of $\sim 100$ au and then increase in separation due to the accretion of high angular momentum material. However, upon examining the evolution of the relatively warm molecular cores in models $\mathrm{H}$ and I (11 and $12 \mathrm{~K}$, respectively) with a larger amplitude of the initial azimuthal density perturbation ( $A=25$ per cent), we find that the overall speed of the evolution of these fragmenting models is much slower than for models A-C. At the end of the simulation, models $\mathrm{H}$ and I have not yet experienced pericentre passage, and the resulting densities and temperatures are lower than for models A-C, as can be seen by comparing the left- and right-hand panels of Figs 5 and 8, respectively. Over the course of the simulation, models $\mathrm{H}$ and I undergo a monotonic decline in eccentricity from $\sim 0.7$ to $\sim 0.5$.

Let us now take a closer look at the evolution of the protostellar discs in models A, B, and C. The time evolution of the Toomre parameter is an important indicator of the growth of gravitational instabilities in the discs which form during the gravitational collapse of our cloud models with values below 1 indicating the onset of 
gravitational instabilities. Figs 9(a)-(e) contain plots of the radial evolution of the Toomre parameter, averaged with respect to the azimuthal angle in the mid-plane of the disc, for models A-E at various instants of time. It can be observed that the time evolution of the Toomre parameter closely follows the development of fragments during the collapse of the cloud models. At the earlier stages of the evolution, all models exhibit gravitational instability within a radial range of $167 \mathrm{au}$. As the clouds evolve further, the gravitational instabilities in models A, B, and C, which start their evolution from temperatures at or below $10 \mathrm{~K}$, gradually sweep larger parts of the discs, almost doubling their active radial range up to $334 \mathrm{au}$. On the other hand, the hotter models D and E do not show this kind of behaviour. For the relatively cold gas models, the part of the initial molecular core which is subject to gravitational fragmentation is about 10 per cent of the initial radius. Beyond this range, the disc seems fairly stable and remains free of fragmentation processes. The time evolution of $Q$ for each model also shows that molecular cloud cores with higher initial temperatures take more time to undergo fragmentation than models which start their evolution with lower initial temperature values. The threshold value of unity at or below which fragmentation should happen never seems to be reached outside of the inner regions by models $\mathrm{D}$ and $\mathrm{E}$, whereas models A-C, which start their evolution from temperatures ranging from 8 to $10 \mathrm{~K}$, show a drop of the $Q$ values well below unity and hence give rise to self-gravitating fragments which are well under way to reach protostellar densities.

We also made an attempt to understand the sensitivity of the structural evolution of collapsing molecular cores on the threshold density that marks the beginning of the adiabatic heating phase. At that moment the collapsing core becomes optically thick enough to trap heat inside leading to a transition from isothermal to adiabatic behaviour of the collapsing gas. For this purpose, we varied the value of the critical density $\rho_{\text {crit }}$ by an order of magnitude around $5 \times 10^{-14} \mathrm{~g} \mathrm{~cm}^{-3}$ and investigated the role of adiabatic heating in determining the evolution of the models. The outcome of this investigation is illustrated by the results for models $\mathrm{F}$ and $\mathrm{G}$, for which the critical density is set to values 10 times lower or 10 times larger than the standard value, respectively. The evolution for model $\mathrm{G}$ is illustrated in Figs 10 and 11. Model G follows a delayed isothermal phase during which a thin but very dense bar is formed connecting the two primary fragments. This bar-like structure subsequently fragments into multiple fragments. This interesting phenomenon also happened in a collapse model test described in Kitsionas \& Whitworth (2002), which even further prolonged the isothermal phase by setting $\rho_{\text {crit }}=5 \times 10^{-12} \mathrm{~g} \mathrm{~cm}^{-3}$. At the later stages of evolution, the secondary fragments combine with each other and produce a third fragment that starts to interact gravitationally with one of the primary fragments hence giving rise to the possibility of a hierarchical system of protobinaries, with one binary as a component of another already formed binary system. This final state of the evolution is shown in panel g6 of Fig. 10, after which we unfortunately had to stop the calculations because of too small time steps. The emerging protobinary system in model $\mathrm{G}$ is found to be the result of a combination of both primary and secondary fragmentation. Note that this evolution is different from that seen in models A, B, C which resulted in protobinary systems consisting only of primary-primary fragments. For comparison, we refer to panels a5, b5, c5, and g6 of Fig. 1 and Fig. 10, respectively. Fig. 12 shows a detailed view of the protobinary system formed as a result of the secondary fragmentation of the bar. The density evolution of this model shows a quicker shift of eight orders of magnitude in density of the fragments that may evolve up to protostellar densities. This evolution happens in roughly half of the time needed for the rest of the models that yielded a binary system to reach densities near $10^{-10} \mathrm{~g} \mathrm{~cm}^{-3}$, at which point hydrogen molecules in the collapsing gas experience molecular dissociation and the second collapse phase starts. The sideway views in Fig. 13 also clearly show the extent of the vertical dimension of the intermediate disc structure, which is found to be much thinner than for any other collapsing model studied in the present work. Although model G could not evolved to the extent of models of A, B, and C, we suggest that the model is well on its way to becoming a stable triple stellar system.

Figs 13 and 14 describe the time evolution of model $\mathrm{F}$ in the equatorial and vertical planes, respectively. The evolution of the core is free of any process of fragmentation for this model. This mainly happens because the inward gravitational pull is challenged by the quicker rise of the temperature of the core so that the gas cannot collapse to the stage where clumps could be formed. Instead, model $\mathrm{F}$ initially collapses to a bar that keeps on gaining thickness and eventually gives birth to a defused spiral structure that surrounds the dense bar as can be seen in panel f6 of Fig. 13. Similarly, we also see in Fig. 14 that such a warm molecular core fails to develop a thin disc structure as in model $\mathrm{G}$.

The evolution of the surface density and Toomre parameter values in models $\mathrm{G}$ and $\mathrm{F}$ are shown in Figs 15 and 16 as well as Figs 17 and 18 , respectively. The surface density evolution seen in models $\mathrm{F}$ and $\mathrm{G}$ is a clear manifestation of how conditions leading to fragmentation play a key role in transferring angular momentum of the disc outwards resulting in infall of gas that gives rise to increased surface densities of the disc and hence a drop in the Toomre parameter values. This inverse relationship between Toomre parameter value and the surface density is obvious when comparing the corresponding panels in Figs 16 and 17 and Figs 17 and 18. Values of the Toomre parameter well above unity also suggest that the thick disc resulting from model $\mathrm{F}$ never experiences a phase of gravitational instability.

A comparison of models $\mathrm{C}, \mathrm{F}, \mathrm{G}$ yields further interesting results on the temperature and density evolution of the molecular core models which are illustrated in Fig. 19. The left- and right-hand panels, respectively, show the evolution of the maximum temperature and density for these three models. It can be seen that changing the critical density over an order of magnitude range results in a direct relation between the critical density and the resulting maximum temperature and density values reached during the evolution with higher critical densities corresponding to higher temperature and density values. This is explained by the fact that a little longer isothermal phase during the collapse allows the molecular gas to get compressed to a denser state, which initially gives rise to a thin filament structure along with multiple fragments which later on in the adiabatic phase become full-fledged protostellar objects. On the other hand, if the adiabatic phase takes over the initial isothermal regime a little earlier, the core might end up as a mere reservoir of gas that never reaches densities corresponding to protostellar systems.

Finally, Fig. 20 shows snapshots of the temperature integrated along the rotational axis for the six models $\mathrm{A}-\mathrm{C}$ (the first row) and for models D, E, and G (second row) at the final state of evolution of the models. Hardly any temperature difference is found for the two fragments reaching the state of protostars in models $\mathrm{A}, \mathrm{B}$, and $\mathrm{C}$. The single protostellar objects arising in models $\mathrm{D}$ and $\mathrm{E}$ correspond to the slightly hotter regions in the two leftmost columns in the second row. However, Model G (the bottom-right panel) represents a significant difference in temperature of the resulting triple system mainly because the system hosts both primary and secondary 


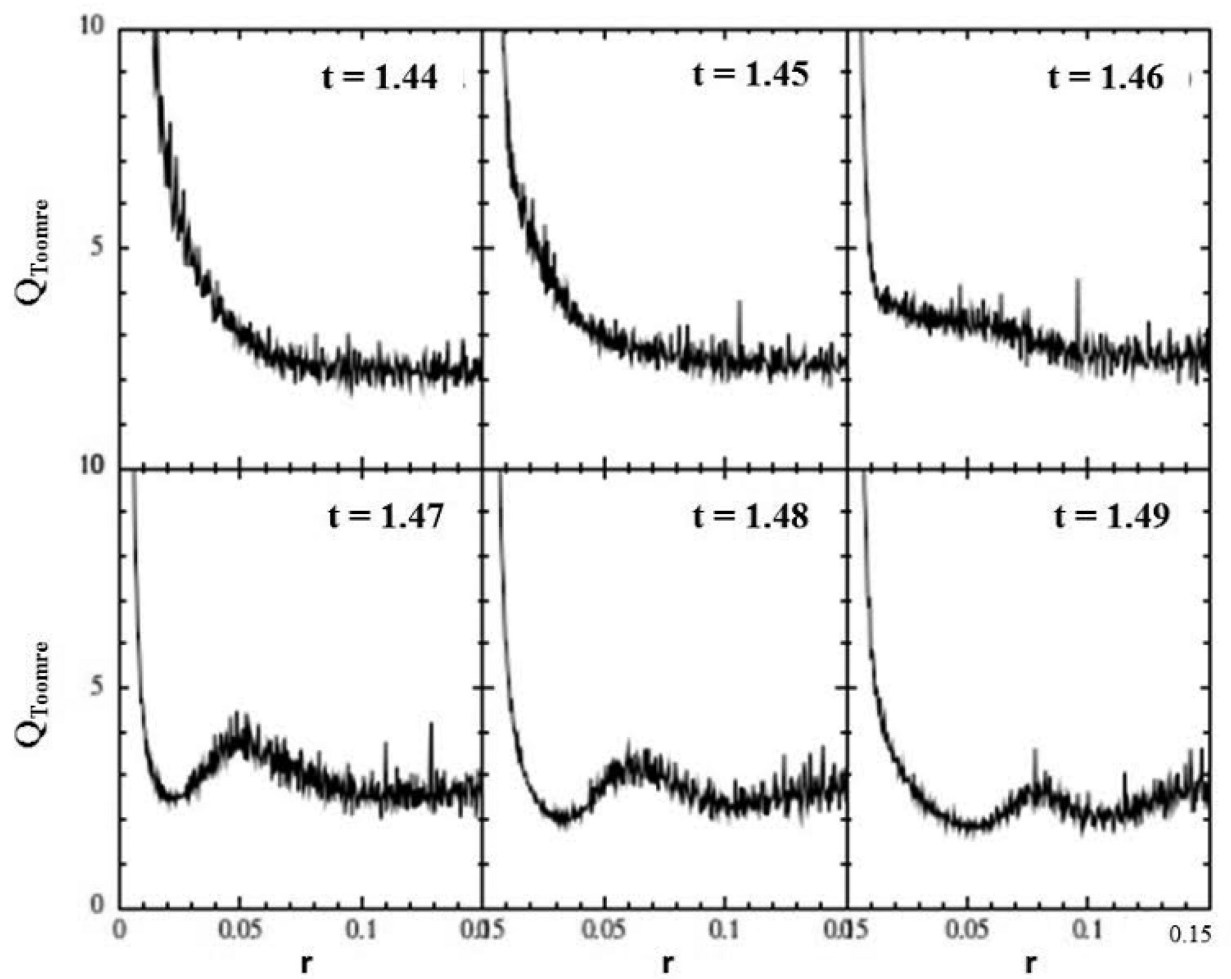

Figure 17. Traces of the Toomre parameter against radial distance $r$ measured in the equatorial plane for model G. The times for each snapshot are given in units of $t_{\mathrm{ff}}$.

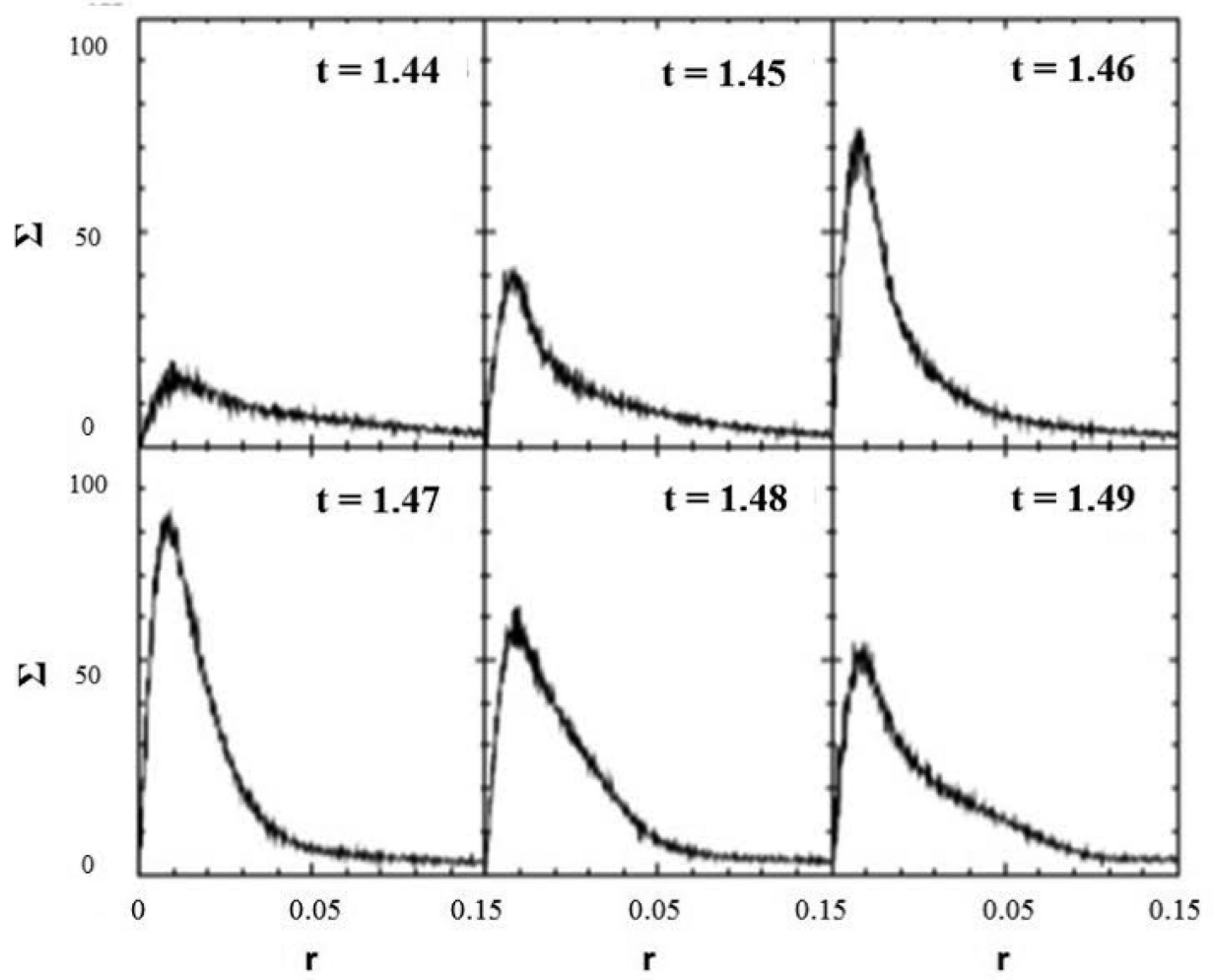

Figure 18. Traces of the surface density against radial distance $r$ measured in the equatorial plane for model G. The times for each snapshot are given in units of $t_{\mathrm{ff}}$. 

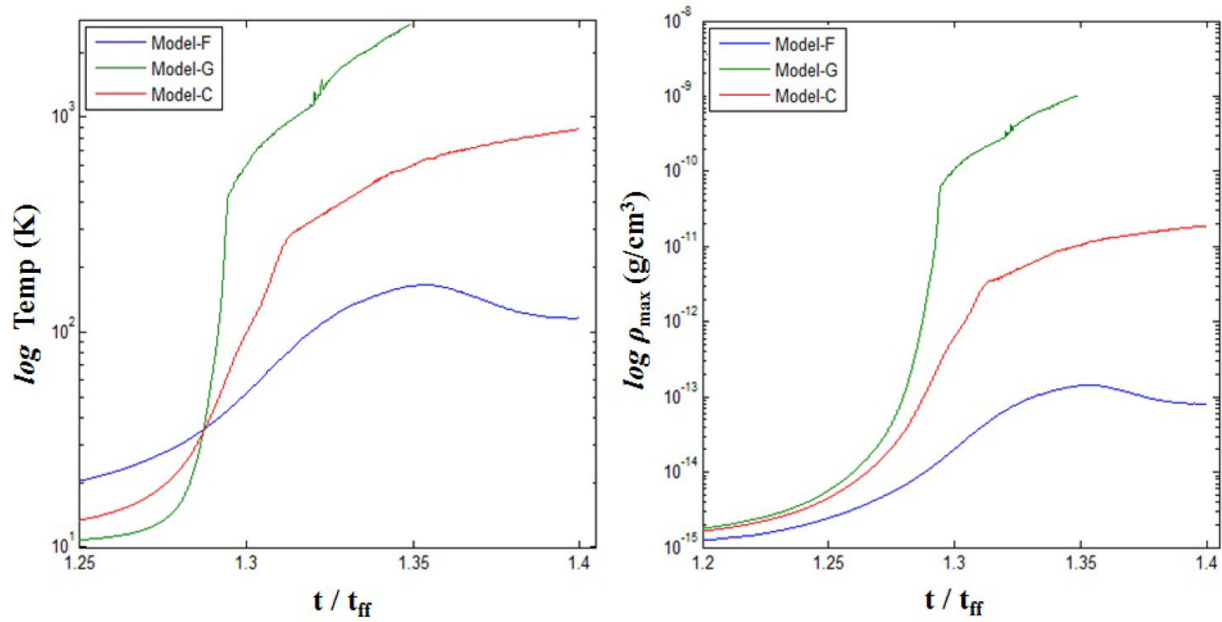

Figure 19. Time evolution of the maximum temperature within the collapsing cloud for models E, F, and G (left-hand panel), and the time evolution of the maximum density for the same set of models (right-hand panel).
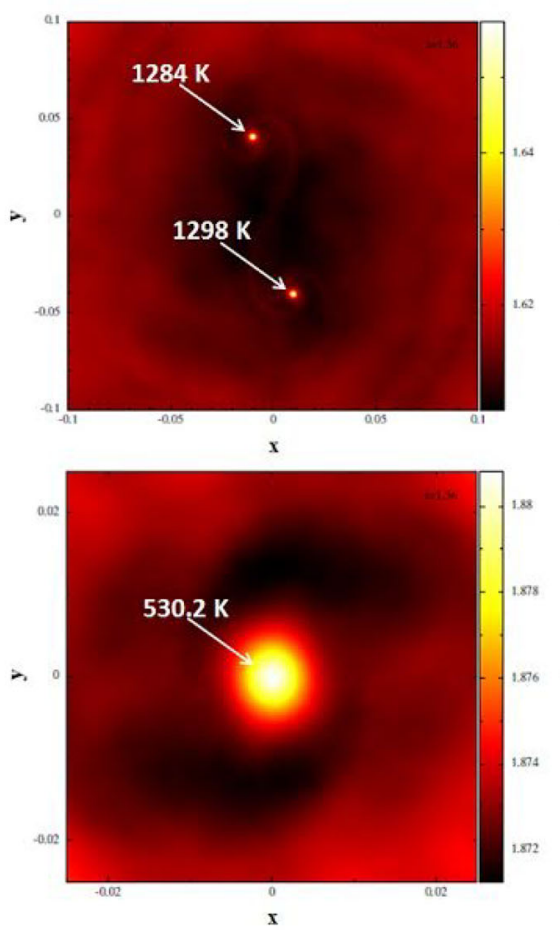



$\mathbf{x}$

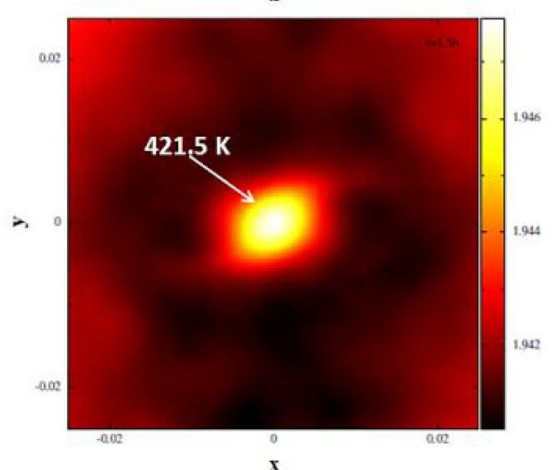

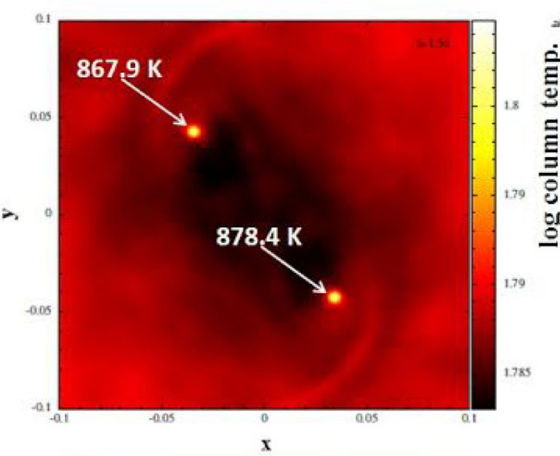

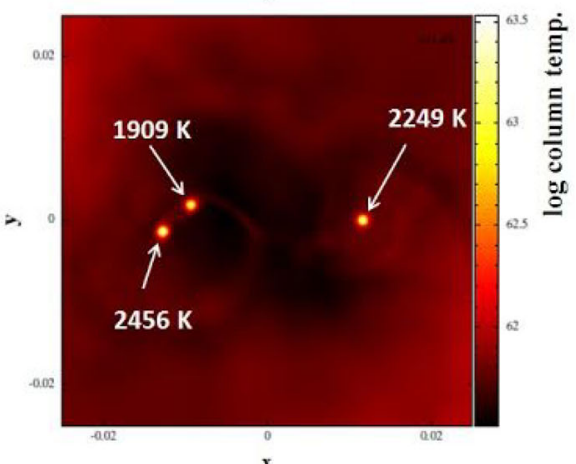

Figure 20. Snapshots of the column temperature integrated along the rotation axis at the final stage of the evolution for models A, B, and C (first row) and for models D, E, and G (second row). The horizontal and vertical dimensions in the $x y$-plane in the first and second row are $0.1 \times 0.1$ and $0.025 \times 0.025$ in dimensionless units, respectively. The colour bar in each panel shows $\log (T)$ in dimensionless units. The values Arrows represent the temperature of the indicated fragment in kelvin.

fragments which have delayed isothermal phases. Moreover, the two primary fragments spend a longer part of their evolution within the isothermal phase compared to the third secondary fragment. This may explain the low temperature which is associated with the secondary fragment compared to the primary fragments in the resulting triple stellar system.

\section{CONCLUSIONS}

In this paper, we have investigated the influence of the initial thermal state of molecular cloud cores on the formation of protostellar binary systems through gravitational fragmentation. We find that in the relatively low-temperature cores in which binaries are formed (with $T_{0}$ in the range $8-10 \mathrm{~K}$ ), the binary separation is a function of the initial thermal conditions prevailing in the molecular cloud cores. Darker and colder molecular cloud cores at $T_{0}=8 \mathrm{~K}$ evolve into protobinary systems with small separation, whereas for slightly higher initial temperatures of 9 or $10 \mathrm{~K}$, an increase in the binary separation is observed based on the results of our models. Our quantitative analysis has therefore revealed a strong thermal sensitivity of the separation of evolving protobinary systems. Molecular cloud cores with temperature above $10 \mathrm{~K}$ and with small amplitude of 
initial azimuthal density perturbation $(A=10$ per cent, models $\mathrm{E}$ and F) do not develop binary systems but form single protostars instead. Further investigation of such cores by introducing a stronger amplitude of perturbation ( $A=25$ per cent), however, has shown that warm cores can indeed be forced to fragment. The evolution of the resulting binaries is apparently much slower than in the colder case, although we have not been able to follow their evolution until after pericentre passage. We also investigated the impact of an effective cooling environment that may prolong the isothermal phase of core collapse. We find that higher values for the critical density which separates the regime of isothermal and adiabatic cloud collapse significantly affects the binary fragmentation process, in that additional secondary fragmentation has been observed in a bar connecting the primary protostellar fragments in the central part of the models. This process happens in a fairly short period of time compared to the freefall time of the cores and can give rise to the formation of multiple hierarchical protostellar systems. On the other hand, a reduction of the critical density, hence a less efficient cooling environment, suppresses fragmentation and may even lead to stable disc structures without star formation in the first place.

\section{ACKNOWLEDGEMENTS}

We thank the Institute of Space and Planetary Astrophysics (ISPA) for financially supporting this research project. We are also grateful to the Pakistani National Center for Physics (NCP) for providing access to the computational facility that was used in this study. We also thank the anonymous referee, whose comments substantially improved the contents of the final version of the manuscript.

\section{REFERENCES}

Arreaga-Garcia G., Klapp J., Sigalotti L. D., Gabbassov R., 2007, ApJ, 666, 290

Bate M., Burkert A., 1997, MNRAS, 288, 1060

Béjar V., Zapatero Osorio M., Perez-Garrido A., Alvarez C., Martin E., Rebolo R., Villo-Perez I., Diaz-Sanchez A., 2008, ApJ, 673, L185

Benson P., Myers P., 1989, ApJS, 71, 89

Boss A., 2001, in Zinnecker H., Mathieu R. D., eds, Proc. IAU Symp. 200, The Formation of Binary Stars. Astron. Soc. Pac., San Francisco, p. 371

Bouy H., Moraux E., Bouvier J., Brandner W., Martin E., Allard F., Baraffe I., Fernandez M., 2006, ApJ, 637, 1056

Burkert A., Bodenheimer P., 1993, MNRAS, 264, 798

Bürzle F., Clark P., Stasyszyn F., Greif T., Dolag K., Klessen R., Nielaba P., 2011, MNRAS, 412, 171

Donati J.-F., Jardine M. M., Gregory S. G., Bouvier J., Dougados C., Mnard F., 2009, EAS Publ. Ser., 39, 133

Duchêne G., Bontemps S., Bouvier J., André P., Djupvik A., Ghez A., 2007, A\&A, 476, 229
Duffin D. F., Pudritz R. E., 2008, MNRAS, 391, 1659

Forgan D., Rice K., 2012, MNRAS, 420, 299

Frank A., Carroll J., Blackman E. G., Heitsch F., Lebedev S., 2013, High Energy Density Phys., 9, 341

Fumitaka N., Li Z.-Y., 2003, ApJ, 594, 363

Gingold R., Monaghan J., 1977, MNRAS, 181, 375

Gomez-Ramirez F., Klapp J., Cervantes-Cota J., Arreaga-Garcia G., Bahena D., 2012, in Klapp J., ed., Experimental and Theoretical Advances in Fluid Dynamics. Springer-Verlag, Berlin, p. 203

Hubber D. A., Batty C. P., McLeod A., Whitworth A. P., 2011, A\&A, 529, 28

Indriolo N., 2011, PhD thesis, Univ. Illinois

Janson M. et al., 2012, in Richards M. T., Hubeny I., eds, Proc. IAU Symp. 282, From Interacting Binaries to Exoplanets: Essential Modeling Tools. Cambridge Univ. Press, Cambridge, p. 460

Kitsionas S., Whitworth A., 2002, MNRAS, 330, 129

Krumholtz M., 2011, in Telles E., Dupke R., Lazzaro D., eds, AIP Conf. Proc. Vol. 1386, XV Special Courses at the National University of Rio de Janeiro. Am. Inst. Phys., New York, p. 9

Launhardt R. et al., 2013, A\&A, 551, A98

Loughnane R. M., Redman M. P., Keto E. R., 2012, in Capuzzo-Dolcetta R., Limongi M., Tornambè A., eds, ASP Conf. Proc. Vol. 453, Advances in Computational Astrophysics: Methods, Tools, and Outcome. Astron. Soc. Pac., San Francisco, p. 33

Luhman K. L., 2004, ApJ, 614, 398

Machida M., Tomisaka K., Matsumoto T., Inutsuka S., 2008, ApJ, 677, 327

Matsunaga H., Inutsuka S., 2000, ApJ, 531, 350

Moeckel N., Bally J., 2007, ApJ, 656, 275

Omukai K., 2000, ApJ, 534, 809

Padovani M., Hennebelle P., Galli D., 2013, A\&A, 560, 13

Peters T., Schleicher D. R. G., Klessen R. S., Banerjee R., Federrath C., Smith R. J., Sur S., 2012, ApJ, 760, L7

Price D., 2007, Publ. Astron. Soc. Aust., 24, 159

Price D., 2012, J. Comput. Phys., 231, 759

Price D., Monaghan J., 2007, MNRAS, 374, 1347

Raghavan D. et al., 2010, ApJS, 190, 1

Stamatellos D., 2007, A\&A, 475, 37

Tobin J. J. et al., 2013, ApJ, 779, 21

Tohline J., 1982, Fundam. Cosm. Phys., 8, 1

Toomre A., 1964, ApJ, 139, 1217

Vanaverbeke S., Keppens R., Poedts S., Boffin H., 2009, Comput. Phys. Commun., 180, 1164

Vanaverbeke S., Keppens R., Poedts S., 2013, Comput. Phys. Commun., 185,1053

Ward-Thompson D., Andr P., Kirk J., 2002, MNRAS, 329, 257

Wiener J., Zweibel E. G., Oh S. P., 2013, ApJ, 767, 8

This paper has been typeset from a $\mathrm{T}_{\mathrm{E}} \mathrm{X} / \mathrm{L} \mathrm{T} \mathrm{E} \mathrm{X}$ file prepared by the author. 